Article

\title{
Textural and Compositional Characterization of Wadi Feiran Deposits, Sinai Peninsula, Egypt, Using Radarsat-1, PALSAR, SRTM and ETM+ Data
}

\author{
Ahmed Gaber ${ }^{1}$, Magaly Koch ${ }^{2, *}$ and Farouk El-Baz ${ }^{2}$ \\ 1 Graduate School of Environmental Studies, Tohoku University, Sendai 980-8576, Japan; \\ E-Mail: agaber@cneas.tohoku.ac.jp \\ 2 Center for Remote Sensing, Boston University, Boston, MA, 02215, USA; E-Mail: farouk@bu.edu \\ * Author to whom correspondence should be addressed; E-Mail: mkoch@bu.edu.
}

Received: 30 October 2009; in revised form: 2 December 2009 / Accepted: 23 December 2009 / Published: 28 December 2009

\begin{abstract}
The present work aims at identifying favorable locations for groundwater resources harvesting and extraction along the Wadi Feiran basin, SW Sinai Peninsula, Egypt, in an effort to facilitate new development projects in this area. Landsat ETM+, Radarsat-1 and PALSAR images of Wadi Feiran basin were used in this work to perform multisource data fusion and texture analysis, in order to classify the wadi deposits based on grain size distribution and predominant rock composition as this information may lead to the location of new groundwater resources. An unsupervised classification was first performed on two sets of fused images (i.e., ETM+/Radarsat-1 and ETM+/PALSAR) resulting in five classes (hybrid classes) describing the main alluvial sediments in the wadi system. Some variations in the spatial distribution of individual classes were observed, due to the different spectral and spatial resolutions of Radarsat-1 (C-band, $12.5 \mathrm{~m}$ ) and PALSAR (L-band, $6.25 \mathrm{~m}$ ) data. Alluvial deposits are mixtures of parent rocks located further upstream often at a great distance. In order to classify the alluvial deposits in terms of individual rock types (endmembers), a spectral linear unmixing of the optical ETM+ image was performed. Subsequently, each class of the fused (hybrid) images was correlated with (1) individual rock type fractions (endmembers) obtained from spectrally unmixing the ETM+ image, (2) the geocoded and calibrated radar images (Radarsat-1 and PALSAR) and, (3) the slope map generated from the SRTM data. The goal was to determine predominant rock composition, mean backscatter and slope values for each of the five hybrid classes. Backscatter coefficient values extracted from both radar data $(\mathrm{C}$ -
\end{abstract}


and L-band) were correlated and checked in the field, confirming that both wavelengths produced more or less similar textural classes that correspond to specific grain or fragment sizes of alluvial deposits. However, comparison of the spatial distribution of matching hybrid classes showed some variations due to the greater discrimination power of surface texture by Radarsat-1 C-band despite its lower spatial resolution. Furthermore, both hybrid classification results showed that regardless of elevation, areas that are covered by fine and moderate grains (fine sand to pebble) and are located along gentle terrains are favorable for groundwater recharge; while areas that are covered by very coarse grains (cobble to boulder) and are located along steep terrains are more likely to be affected by flash floods.

Keywords: optical-microwave data fusion; textural classification; spectral unmixing; wadi deposits; water resources; flash floods; Sinai Peninsula; Egypt

\section{Introduction}

Egypt has a rapidly growing population that now exceeds 76 million. Most of the population is concentrated in less than 5\% of its land area along the Nile River and Delta. Furthermore, food, water and housing demands are expected to increase. Such conditions necessitate the development of unused areas fringing the Nile valley and in the Sinai Peninsula. Although many industrial, commercial, agricultural and tourism projects have been initiated and accomplished along the northern and southern coasts of Sinai, the Wadi Feiran basin remains underdeveloped. The lack of water resources necessary for initiating any development plans is the main reason behind the delay in developing this area.

The Wadi Feiran basin is located in the southwestern part of the Sinai Peninsula, Egypt, and covers an area of about $1,851.5 \mathrm{~km}^{2}$. It forms part of a rugged mountain range of igneous and metamorphic rocks, which dominates South Sinai region; thus the basin is predominantly composed of PreCambrian granitic (old and young), metamorphic, volcanic and sedimentary rocks (Figure 1). Wadi Feiran basin is dissected by a number of wadis, which drain towards the Gulf of Suez in the west, e.g., Feiran, El-Akhdar, El-Sheikh and Solaf. Climatically, the region is typically arid; precipitation is scarce and random, and temperatures are high during most of the year. The little rain that does occur falls in winter, when temperatures are relatively low. The distribution of the scant precipitation appears to be mainly controlled by topography. Thus, the low coastal areas seldom get more than $15 \mathrm{~mm}$ of rain per year. The higher regions, such as Gebel Katherina in the east, receive approximately 50 $\mathrm{mm} /$ year of precipitation, partly as snow [1]. During exceptional years, torrential rains can raise the annual precipitation total to more than $100 \mathrm{~mm}$.

El-Baz and Himida [2] stated that the wadi fills constitute the best potential of low-cost groundwater resources in the southern Sinai mountain region. In some cases dikes serve as natural dams that hold water upstream in the valley fill such as in the case of Wadi Feiran. Gaber et al. [3] demonstrated that two paleolakes formed along the Wadi Feiran basin after damming the torrential rainfall, during humid periods, by the last invading acidic dikes. Such paleolakes were loaded with fine sediments, which were later deposited as well-stratified thin beds and formed the current outcropping 
lacustrine deposits. The same authors suggested that wherever the lacustrine sediments cropped out the underlying wadi deposits most probably stored water.

Figure 1. Simplified geologic map of the Wadi Feiran Basin, southwest Sinai, (CONOCO, 1987).

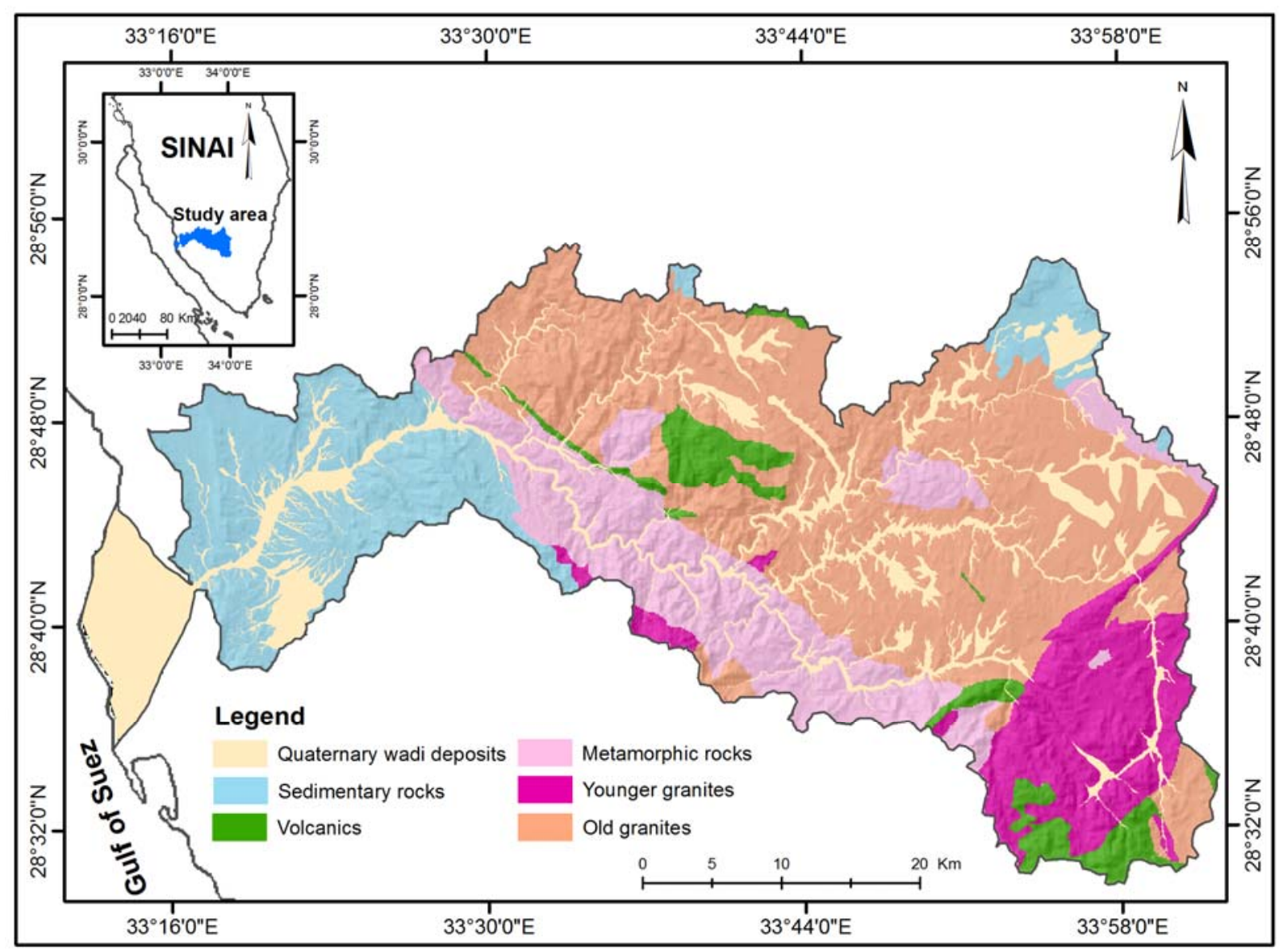

New satellite systems operating in the optical and microwave region of the spectrum are currently providing a continuous stream of improved and updated information on land surface features as well as terrain characteristics. Furthermore, satellite images can be used to extract information about the distribution of textural and compositional characteristics of wadi course materials. In this work, images from four different sensors, namely one optical (Landsat ETM+) and three microwave sensors (SRTM, Radarsat-1 and ALOS-PALSAR) were used to delineate favorable areas for groundwater recharge as well as areas of increased flash flood hazard risks in the Feiran basin.

This work builds upon previous research conducted by Koch et al. [4] and Koch and El-Baz [5] in the Samail basin of the Oman Mountains, where a new approach for mapping and classifying active wadi systems in arid environments was first developed using Landsat TM data in conjunction with Radarsat-1 imagery. The aforementioned authors found that the combination of complementary data from optical and microwave sensors enabled them to better identify relative stream activities (i.e., erosive power) of wadi systems in arid environments. This was done based on: (a) determination of spectral and textural properties of the alluvial wadi floor materials (by means of optical and microwave data fusion), (b) detection of variations in surface roughness as related to lithology and 
weathering products, (c) classification of wadi floor materials in terms of their grain size and mineralogy, and (d) identification of rock types contributing to mineral composition of individual wadis (by means of spectral unmixing) and identification/location of source-rock area. These wadi system characteristics provide useful information on their efficiency in carrying bed load sediments and, thus, surface flow.

The present work is a refinement of the methodology first applied by Koch et al. [4] and Koch and El-Baz [5] in the Oman Mountains. It combines both the optical-microwave data fusion and pixel unmixing techniques utilized in the previous works to extract the textural and compositional characteristics of wadi deposits, and adds a third and new component which is the slope (terrain characteristics). Furthermore, three types of microwave data were used in this work, i.e., SRTM DEM for extracting terrain characteristics, and Radarsat-1 and PALSAR for data fusion and textural classification of wadi deposits. PALSAR data were used for field validation due to their more recent acquisition date. The different frequencies of Radarsat-1 (C-band) and PALSAR (L-band) were compared and evaluated in terms of surface roughness estimations. The optical-microwave data fusion was performed on two data sets, i.e., ETM + and Radarsat- 1 and ETM + and PALSAR. The classification results were checked in the field and individual classes were labeled according to wadi deposit type and size (texture), as well as wadi surface slope in order to produce maps of: (1) favorable areas for groundwater recharge and, (2) increased flash flood hazard risks in the event of a rainstorm. These maps provide much needed information for decision makers to initiate sustainable development of Wadi Feiran basin.

\section{Data and Methods}

\subsection{Dataset Characteristics}

Images from four sensors were utilized in this work. Two sets of orthorectified Landsat ETM+ scenes acquired on 22-Dec-2000/10-Sep-2000 and 21-Feb-2008/15-Mar-2008 represent the optical data, of which the first six reflective bands (1-5 and 7) with a spatial resolution of $28.5 \mathrm{~m}$ and the panchromatic band 8 with a $14.25 \mathrm{~m}$ pixel resolution were used. In the first set one single scene (p175r040; 22-Dec-2000) covers almost the entire Wadi Feiran basin; however, the south-easternmost tip of the basin needed to be completed with the second scene (p174r040; 10-Sep-2000). In the second set (p174r040; 21-Feb-2008 and p175r040; 15-Mar-2008) both scenes show strips with no data due to the malfunctioning of the scan line corrector (SLC). Also their scene footprints are slightly different from the first set requiring a larger portion of the second scene in order to cover the entire basin area. The microwave dataset is composed of (1) a Shuttle Radar Topography Mission (SRTM) digital elevation model (DEM) with an original resolution of $30 \mathrm{~m}$, downgraded to $90 \mathrm{~m}$ resolution and datavoids filled, (2) a Radarsat-1 (C-band, HH-polarization, descending orbit) Synthetic Aperture Radar (SAR) scene with a pixel size of $12.5 \mathrm{~m} \times 12.5 \mathrm{~m}$ and an incidence angle of $35.085^{\circ}$, acquired on 18 Nov-2000, and (3) two ALOS PALSAR (L-band, HH-polarization, ascending orbit) scenes acquired on 26-Jan-2008, and 24-Feb-2008, with a spatial resolution of $6.25 \mathrm{~m}$ and an incidence angle of $34.3^{\circ}$. Due to the smaller footprint of the fine resolution PALSAR scenes $\left(70 \times 60 \mathrm{~km}^{2}\right)$ a total of two scenes were needed to cover the entire Wadi Ferain basin. In the case of the Landsat ETM+ 2000 set and Radarsat-1 basically one scene of each was enough, as the ETM + covers $95 \%$ and the Radarsat- 1 
covers the entire study area. In addition, a geologic map at the scale of 1:500,000 [6] and high resolution images (IKONOS) viewable in Google Earth were utilized as ancillary data in this work.

\subsection{Image Processing Methods}

Two standard image processing methods were applied in classifying the deposits in Wadi Feiran, namely data fusion and linear mixture modeling. Both methods represent well established and widely used image processing techniques, however, their combined use in arid land applications is still rare, especially with broad band data. Although other more sophisticated textural (e.g., gray-level co-occurrence matrix) and spectral (e.g., multi-layer feed-forward neural network) analysis methods exist [7], one objective of this work is to demonstrate a relatively simple and straightforward methodology to classify highly heterogeneous geological deposits. The first method was used to merge microwave and optical data to achieve a better textural and spectral classification of the wadi deposits. The second method was used to determine the predominant mineral composition of the resulting classes. The reason for selecting and combining both methods is that this way the nature and source areas of the wadi deposits could be determined based not only on mineral composition (spectral classification) but also soil/rock fragment size (textural classification), so that a relationship can be established between wadi deposits and stream power, as well as wadi deposits and upstream source-rock area. The first relationship could reveal which processes are more active, whether erosive or depositional, and where do they predominantly occur along the wadi systems. The second relationship could reveal the location of the source areas of the rock fragments found in the wadi beds and their main transportation routes. The underlying idea is that both relationships may be used to identify basin areas that produce most of the runoff and are more efficient in eroding and transporting sediments to the alluvial fans at the basin outlet. In addition, sediment types and grain sizes may reveal their depositional environment, i.e., whether they were deposited under turbulent or laminar surface flow conditions. Understanding these relationships could ultimately lead to (1) better prediction of flash flood prone areas and (2) location of potential groundwater recharge and accumulation areas.

\subsubsection{Multisource Data Preparation and Fusion}

Multisource data fusion was performed using same year and same season optical and microwave data sets, i.e., Landsat ETM+ with Radarsat-1 data of December 2000 and Landsat ETM+ with PALSAR data of January/March 2008. However, the Landsat ETM+ and Radarsat-1 data fusion had the advantage that: (1) they both cover more or less the entire Wadi Ferain basin with one scene respectively (mosaicking of a small portion was only required for the ETM + data to complete the south-easternmost tip of the basin), thus, minimizing misregistration errors; and (2) the shorter wavelength of $\mathrm{C}$-band would be more sensitive to subtle changes in wadi surface roughness (micro-roughness) than the L-band with its longer wavelength and greater penetration depth. This latter aspect was analyzed by comparing the Radarsat-1 and PALSAR backscatter coefficient values of individual wadi deposits (in terms of predominant lithology and grain size) and corroborating them with field observations. The field campaign was carried out in February 2008 approximately at the time when the Landsat ETM+ and PALSAR data were acquired. 
Figure 2. Fused images of Landsat ETM+ (bands 7, 4, 2 as RGB) (a) with Radarsat-1 (2000) and (b) PALSAR (2008) data. Note that the 2008 ETM+ scenes have the SLC off.

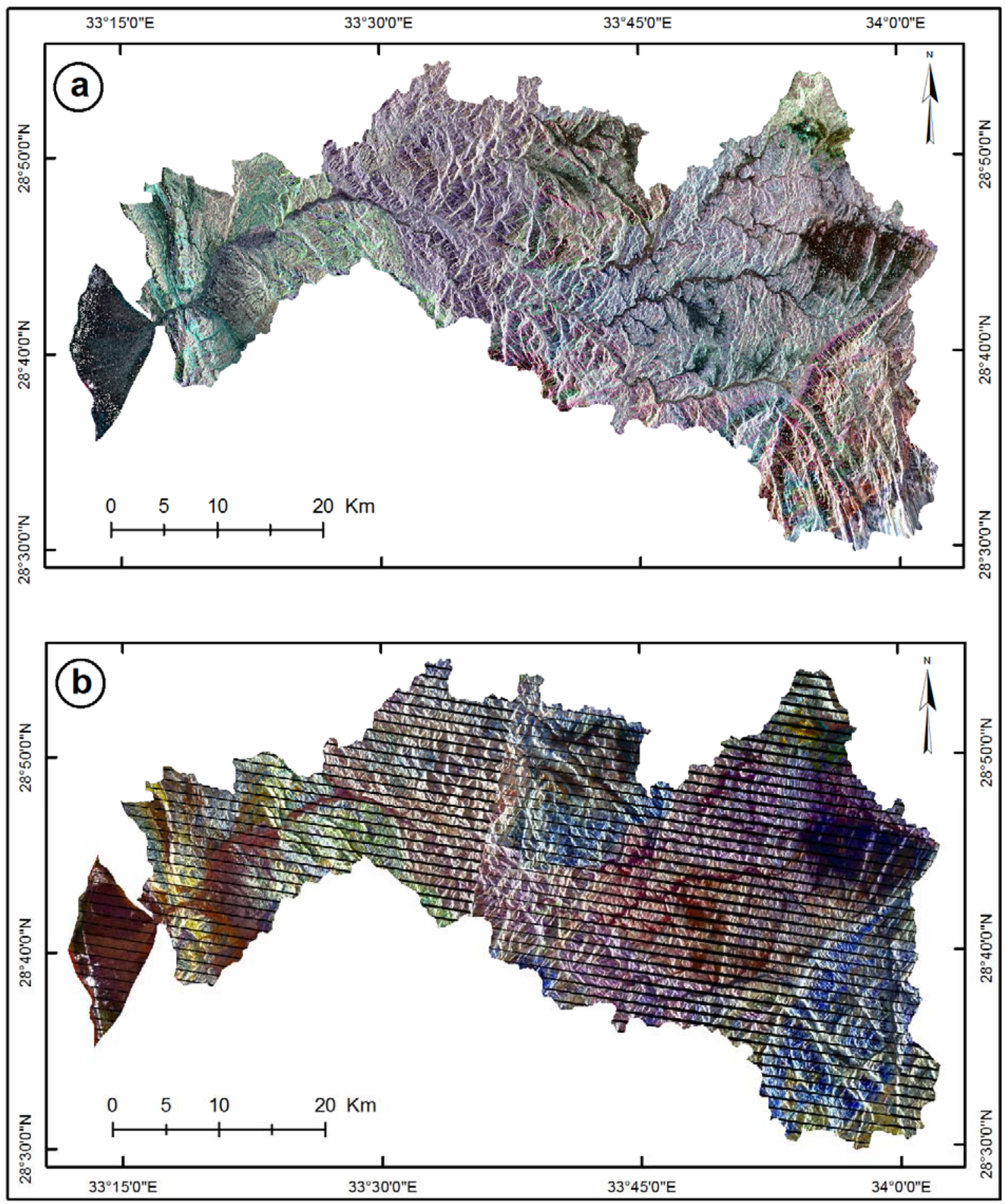

The data fusion method was performed by applying a Principal Component (PC) spectral sharpening algorithm [8] that is implemented in ENVI image processing software [9]. This is a popular data fusion method that is normally used to sharpen a low resolution multi-band image with a high resolution panchromatic band. However, it can also be used for fusing optical and microwave data [10]. The following steps were performed:

1. Speckle reduction of the SAR image using a Lee-enhanced filter, 
2. Data scaling of the SAR image from 16 bit to 8 bit,

3. Georeferencing of the SAR and Landsat ETM+ images to a common UTM coordinate system,

4. Performing a PC transformation to Landsat ETM+ bands $1-5$ and 7,

5. Replacing PC1 with the SAR image as the high resolution component,

6. Performing an inverse transformation resulting in fused SAR/ETM+ bands $1-5$ and 7.

These steps were applied to both SAR data sets, i.e., Radarsat-1 and PALSAR. In both cases a Lee-enhanced filter with a kernel size of $5 \times 5$ was applied to the raw SAR image before 16 to 8-bit data conversion and geocoding. By filtering the 16-bit data instead of the 8-bit data less information is lost (when using the smaller 8-bit data range) while improving the appearance of the image for subsequent ground control point selection [11]. Furthermore, the Lee filter preserves mean backscatter values especially of homogeneous areas (e.g., smooth alluvial fan materials) by reducing the inherent speckle in SAR images [12]. The control points were selected only in the flat alluvial areas (wadi floors) because no topographic distortion correction was performed to the SAR images to compensate for foreshortening, layover, and shadow effects in mountainous areas. The SAR images were resampled to the same pixel size as the ETM+ band $8(14.25 \mathrm{~m})$ image using 35 (Radarsat-1) and 48 (PALSAR) well distributed control points, and a second order polynomial function giving a RMS error of less than 0.5 pixels ( 0.47 for Radarsat- 1 and 0.187 for PALSAR). Previous to the data fusion, the first six ETM+ bands (1-5 and 7) were sharpened using band 8 and the Gramm-Schmidt Sharpening method in ENVI [9].

Color composites of the fused SAR-ETM+ bands 7, 4, 2 displayed as RGB render the best color contrast and are shown in Figure 2. The alluvial areas on the resulting ETM+/Radarsat-1 and ETM+/PALSAR hybrid images show improved contrasting features, meaning that better classification results can be achieved for these areas than when using only optical or microwave data alone. This observation is in line with a study conducted by Koch and El-Baz [5] in Samail basin, Oman, where they compared classification results of wadi deposits using solely Landsat TM versus TM/Radarsat-1 hybrid data. They then compared the classification results to detailed cartographic information on the geology and soils and concluded that the TM/Radarsat-1 classification proved to be superior to the TM classification in terms of discriminating wadi surface materials.

In the present work a 1:500,000 geological map of Wadi Feiran basin was scanned, digitized and used as a reference. In addition, a detailed drainage network map was prepared by digitizing wadi courses from high spatial resolution images (IKONOS) available in Google-Earth. Both digitized maps, i.e., the geological units and alluvial wadis, were later used for creating masks to perform image processing steps on selected areas, as outlined below.

\subsubsection{Classification of Optical/Microwave Hybrid Images}

An unsupervised classification using a K-means clustering algorithm (with 10 initial classes and five iterations) was performed on the alluvial areas of Wadi Feiran basin using both the ETM+/Radarsat-1 and the ETM+/PALSAR hybrid images (with respectively six bands) as input data. The choice of classification procedure was based on the fact that wadi deposits are spectrally and texturally very diverse as they are made up of a whole range of rock types and fragment sizes. 
Figure 3. Unsupervised classification of the wadi alluvium using two sets of fused optical/microwave images: (a) ETM+/Radarsat-1 of 2000, and (b) ETM+/Palsar of 2008. Enlarged sections (A and B) highlight class distribution differences.

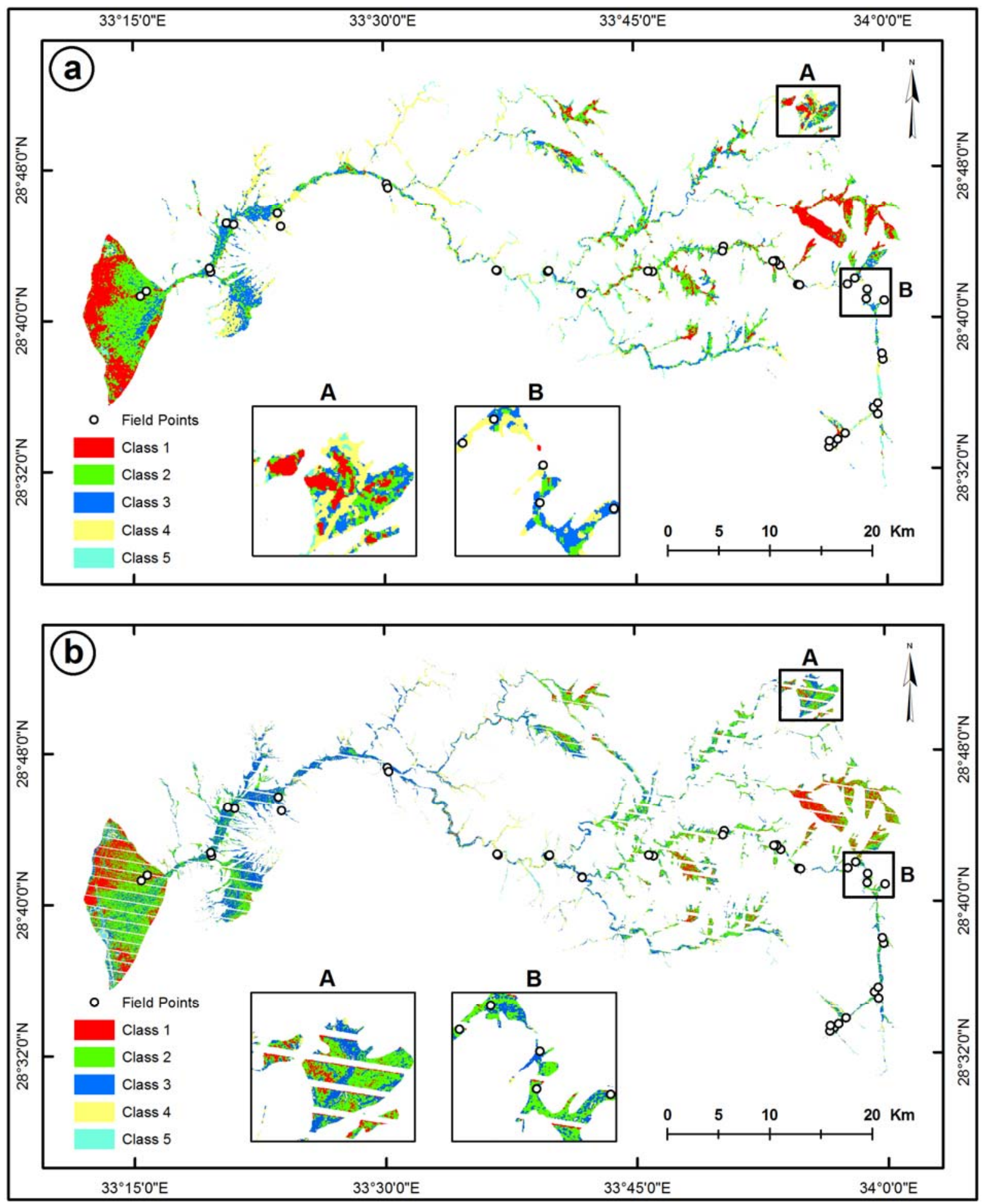

Unsupervised classifiers are especially appropriate for exploring the natural clusters in a data set, and a good method to determine spectral and textural discrimination power of the fused data set. Prior to running the classifications a vegetation mask was applied using the soil-adjusted-vegetation-index (SAVI) to restrict the classification to alluvial areas. Restricting the image data to the area of interest 
helped to reduce the number of output classes to five, which greatly improved their interpretability. In addition, the application of a majority filter with a $5 \times 5$ kernel size to the ETM+/Radarsat-1 and ETM+/PALSAR classified images improved the results by removing noise (spurious class pixels due to radar speckle) from the data, thus, reducing the variance within the resulting clusters (Figure 3). The classes are the result of a combination of spectral and textural properties of the wadi deposits. Before assigning them a final label they were checked in the field and correlated with slope characteristics and the unmixing results described below to determine their probable nature.

\subsubsection{Spectral Unmixing of the ETM+ Image}

Recent advances in image processing software have led to the development of new algorithms operating at sub-pixel resolution, including spectral mixture modeling. Originally developed for hyperspectral data and later applied to multispectral data, this method has been successfully used for mineral mapping $[13,14]$ and land degradation studies in semi-arid areas $[15,16]$. In this paper, spectral unmixing was chosen as the most appropriate method to describe the spatial variability of alluvial areas in Wadi Feiran basin. It is postulated that the alluvial fills of the wadi systems are composed of spatially varying proportions of upstream source rocks. Therefore, spectral mixture modeling may enable tracing the predominant rock components of the wadi fills from the alluvial fan area back to their source area upstream. This might provide useful information about the erosive power and activity of individual wadis and the depositional conditions of their bed load materials.

As stated above, the Landsat ETM+ image of December 2000 covers almost the entire study area and was obtained prior to the SLC malfunctioning. Therefore, this scene was used for the spectral unmixing procedure. It was first geometrically and atmospherically corrected (using the dark object subtraction method) before converting the raw data to radiance values. Image endmembers were selected based on a three-step procedure. First, the Minimum Noise Fraction (MNF) transformation [17] was applied to the six reflective ETM + bands (1-5 and 7) to determine their inherent dimensionality and to separate the noise fraction from the true signal. Next, a Pixel Purity Index (PPI) function was applied to the MNF bands, after discarding the lowest-order component which contains mostly noise. The PPI function is used to locate the most spectrally extreme pixels that typically correspond to mixing endmembers [18].

In order to facilitate the selection of endmembers, the PPI image was divided into five PPI sub-areas to reduce the number of potential endmembers contained in the subareas. The five PPI sub-areas represent the main rock units found in Wadi Feiran basin as shown in a generalized version of the 1:500,000 geological map of the study area [6]. This generalization was achieved by grouping similar lithological units into five major rock types, namely old granite, young granite, metamorphic, volcanic and sedimentary rocks (Figure 1), and were used for masking the PPI sub-areas. A threshold was then applied to each PPI sub-image to highlight pixels that were recorded as statistically extreme, thus, representing spectrally pure endmembers. Finally, these pixels were used in conjunction with the MNF data in an interactive n-dimensional visualization procedure [9] to locate, identify and cluster the purest pixels with the most contrasting spectral responses in the image data. This procedure was applied to each of the five rock units. 


\subsubsection{Endmember Selection}

The above procedure resulted in the extraction of 20 image endmembers. This number was reduced to five in order to run the spectral unmixing model, due to the low dimensionality of ETM+ bands and model requirements. Inspection of the spectral signature of the 20 endmembers and their geographic location within the image, as well as several preliminary unmixing results using different endmember combinations, led to the selection of five representative endmembers (one for each major rock type), excluding shadows and vegetations (Figure 4). These endmembers were then used as inputs in a partially unconstrained unmixing procedure. The resulting root-mean-square (RMS) error image gave the lowest value range for this set of endmembers, confirming that they represent the best endmember combination out of several possibilities.

Figure 4. Spectral signature of the five final image endmembers.

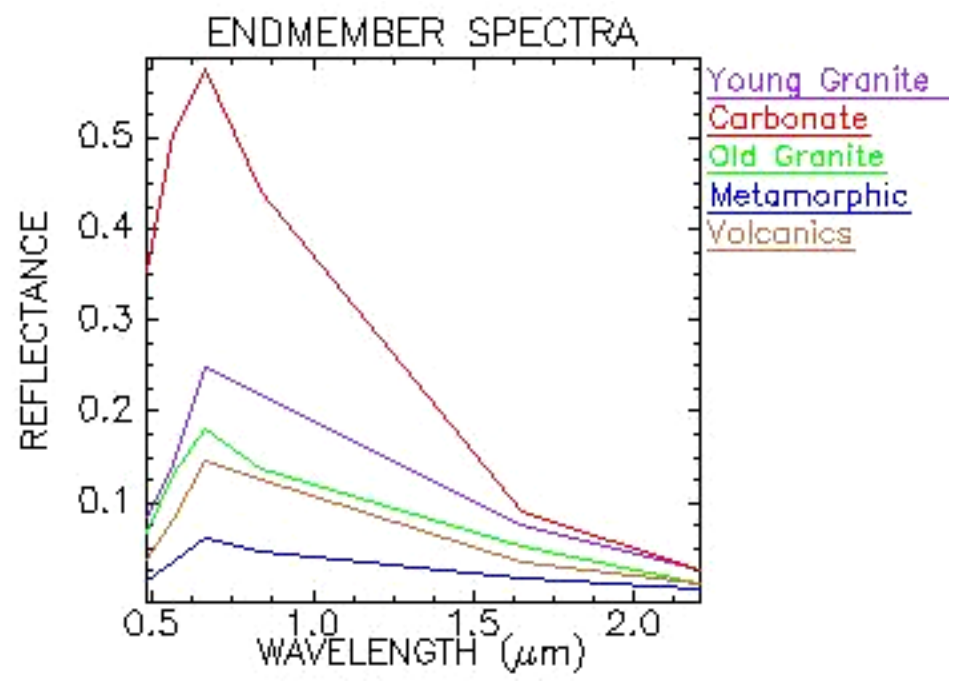

\subsection{Correlation Analysis}

The SRTM DEM was used to generate the slope map (Figure 5) in order to correlate its information with the five classes of the hybrid images (Figure 3) using the Zonal Statistics as Table function in ArcGIS 9.2. This function correlates values of a raster within the zones of another dataset and outputs the results in a table. The resulting table was plotted as a graph using Excel program and is further described in the next section below. The same ArcGIS function was used to correlate the five classes of the classified hybrid images with the endmember unmixing results (fraction images) to determine the predominant rock composition within each class (Figure 6). Furthermore, the classification results and the slope map were correlated to average backscatter values of Radarsat-C band and PALSAR-L band at 41 selected points that were visited in the field in February 2008. The objective was to find out whether significant correlations exist between terrain slope, predominant rock composition and fragment or grain size of wadi deposits, and whether C and L bands are equally capable of detecting subtle textural changes in wadi bed-load size. The correlation analysis results are discussed below. 
Figure 5. Slope map as percent derived from SRTM $90 \mathrm{~m}$ resolution data.

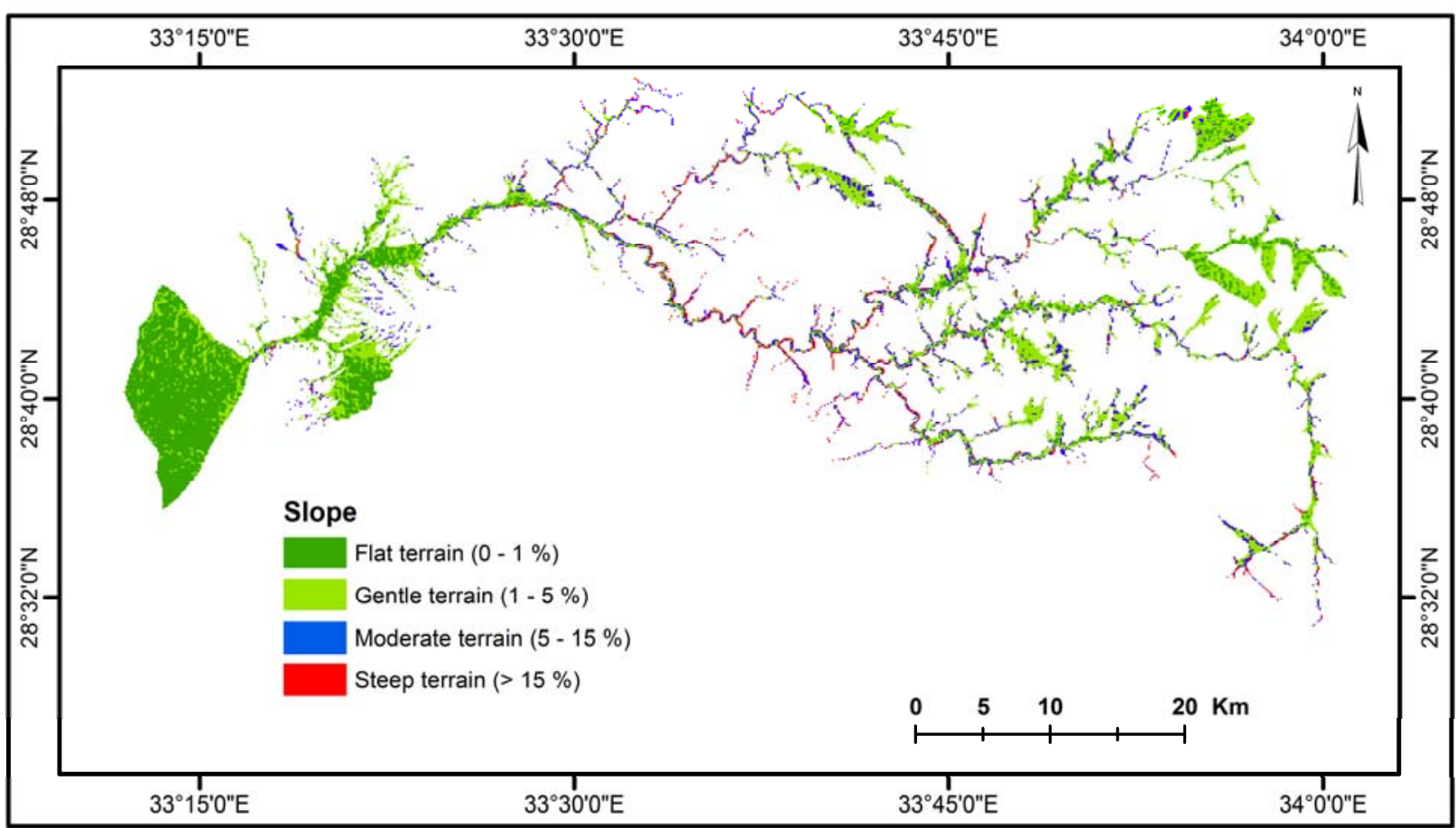

\section{Results and Discussion}

\subsection{Correlation between Hybrid Classes, Backscatter and Slope}

The five resulting classes of the ETM+/Radarsat-1 and ETM+/PALSAR fused images were checked in the field in order to determine their nature (Figure 3). It was concluded that the clusters in the classified hybrid image respond primarily to the amount of backscatter (due to the SAR component) and to a lesser extent to variations in hue and saturation (due to the ETM+ component). The colors assigned to the individual classes in Figure 3 are: class 1 (red), class 2 (green), class 3 (blue), class 4 (yellow) and class 5 (cyan). Each of the classes was overlain on previously geocoded and calibrated Radarsat- 1 and PALSAR data and used to determine the range of backscatter coefficient values (sigma expressed in decibels $(\mathrm{dB})$ ) and their respective range/mean/standard deviation corresponding to each class (Table 1). The SAR calibration was performed using commercial software [9] for the Radarsat-1 data and a public domain software [19] for the PALSAR data. In both cases the original 16-bit raw data was converted to radar backscatter coefficient sigma nought $(\sigma 0)$ using the SRTM DEM for calculating the local incidence angle. Calibration followed by geocoding of the raw SAR data was a necessary step in this part of the analysis since both data sets were to be compared to each other and correlated with field information. Table 1 shows that the median $\sigma 0$ values of Radarsat-1 and PALSAR increase from class 1(red) to class 5 (cyan); however, their values differ as PALSAR shows consistently lower (weaker) backscatter values than Radarsat-1 (Table 2). In addition, the five classes from the unsupervised classification fall within well-defined data ranges that show overlapping class boundaries but have very distinct mean values. The data range overlap is mainly due to the fact that classes represent not only textural but also spectral information, and are, thus, hybrid classes. 
Figure 6. Correlation between the output classes of ETM+/Radarsat-1 hybrid image and slope (a), and fraction maps of image endmembers (b, c, d, e, and f).
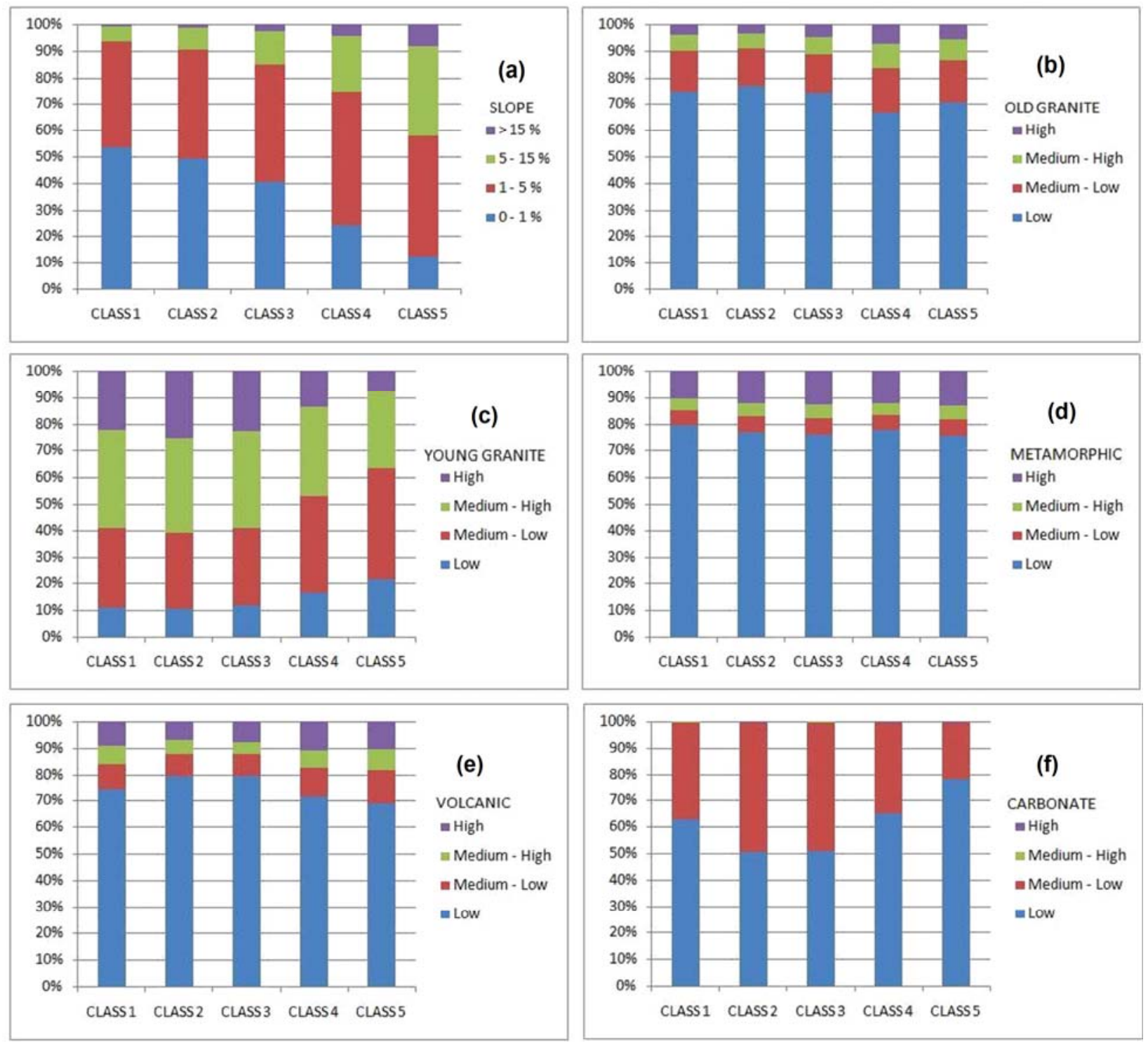

The results in Tables 1 and 2 suggest that class 5 (cyan) represents surface types with the highest surface roughness and thus the strongest backscatter signal (i.e., wadi deposits with very coarse grains and rock fragments) than any of the other four classes. On the other hand, class 1 (red) represents the smoothest surface type (very fine grains) within the alluvial areas of Wadi Feiran basin and shows generally weak backscatter signals (low $\sigma 0$ values) in both radar data sets. Class 2 (green), class 3 (blue) and class 4 (yellow) represent surfaces with gradually increasing roughness or backscatter coefficient values and are situated between the smoothest class 1 and the roughest class 5 (Table 2). Spatially, the distribution of the five classes does not show any significant correlation with elevation, meaning no clear spatial pattern can be recognized along the basin from its upstream portion in Saint Katrine (east) to its downstream outlet in Gulf of Suez (west), except for class 5 which is persistently found along short, steep tributaries and urban areas. However, there seems to be some spatial 
correlation between the hybrid image classes (Figure 3) and slope (Figure 5) with higher class numbers tending to occur at steeper slopes (further explored later in the text).

Table 1. Backscatter statistics (in $\mathrm{dB}$ ) of Radarsat-1 (C-band) and PALSAR (L-band) data for each of the classes produced by unsupervised classification of the respective hybrid images.

\begin{tabular}{lllllll}
\hline \multicolumn{1}{c}{ CLASSES } & \multicolumn{3}{c}{ RADARSAT-1 } & \multicolumn{3}{c}{ PALSAR } \\
\hline & Range & Mean & STD & Range & Mean & STD \\
\hline Class1 (red) & -16.2 to -10.2 & -13.11 & 2.68 & -22.0 to -17.6 & -19.81 & 1.40 \\
Class2 (green) & -13.9 to -8.2 & -10.95 & 2.46 & -19.6 to -15.2 & -17.32 & 1.38 \\
Class3 (blue) & -12.3 to -6.4 & -9.25 & 2.50 & -16.3 to -11.4 & -13.87 & 1.50 \\
Class4 & -11.2 to -4.1 & -7.46 & 2.94 & -12.38 to -5.77 & -9.30 & 2.04 \\
(yellow) & & -5.52 & 4.52 & -6.14 to 6.37 & -4.04 & 3.90 \\
Class5 (cyan) & -10.9 to 0.8 & - & & & & \\
\hline
\end{tabular}

Results of this first correlation analysis confirm that the unsupervised classifications of both hybrid images are largely influenced by the contribution of the microwave component and to a lesser degree by the optical component. Moreover, the ranges of backscatter coefficient values of Radarsat-1 and PALSAR are positively correlated as shown in Figure 7 where mean values where extracted at 41 selected sites visited in the field (Figures 3 and 8). This means that both microwave images of Radarsat-1 and PALSAR produced classification results where the individual classes do correspond to significant changes in grain sizes of wadi deposits as corroborated by field investigation (Figure 8).

Table 2. Mean backscatter coefficient values (in $\mathrm{dB}$ ) of Radarsat-1 and PALSAR data for each of the classes with corresponding roughness/grain size as observed in the field.

\begin{tabular}{llll}
\hline \multicolumn{1}{c}{ Class (color) } & \multicolumn{2}{c}{ Backscatter (mean) } & Roughness and grain size \\
\hline & RSAT-1 & PALSAR & \\
\hline Class 1 (Red) & -13.11 & -19.81 & Smooth (sand, pebble) \\
Class 2 (Green) & -10.95 & -17.32 & \\
Class 3 (Blue) & -9.25 & -13.87 & To \\
Class 4 (Yellow) & -7.46 & -9.30 & \\
Class 5 (Cyan) & -5.52 & -4.04 & Rough (cobble, boulder) \\
\hline
\end{tabular}

Furthermore, the consistently stronger backscatter signal (higher $\sigma 0$ values) of Radarsat-1 with respect to PALSAR are primarily attributed to differences in backscatter amount. In arid environments, the backscatter values are mainly a function of surface roughness, wavelength of the radar pulse, and dimensions of the target in relation to the radar pulse [20]. Whether one and the same surface feature is seen as smooth, intermediate or rough in a radar image depends on the radar beam depression angle and radar wavelength [10]. Therefore, Radarsat-1 data produce higher backscatter values than PALSAR for the same target as both have similar depression angles but different wavelengths with the shorter C-band being more sensitive to surface micro-relieve than the longer L-band [21]. Comparison of the backscatter statistics of individual classes produced by the two sets of hybrid images with field 
observations confirms that Radarsat-1 data show higher median values than PALSAR data for equivalent classes (Table 2 and Figure 8). This suggests that the differences in data ranges between the calibrated Radarsat-1 and PALSAR data had little effect on the outputs of the classifier, which nevertheless produced comparable classes in terms of grain/fragment sizes as checked in the field.

Figure 7. Correlation of mean backscatter coefficient values (in $\mathrm{dB}$ ) of Radasat-1 and PALSAR at surveyed field locations along Wadi Feiran.

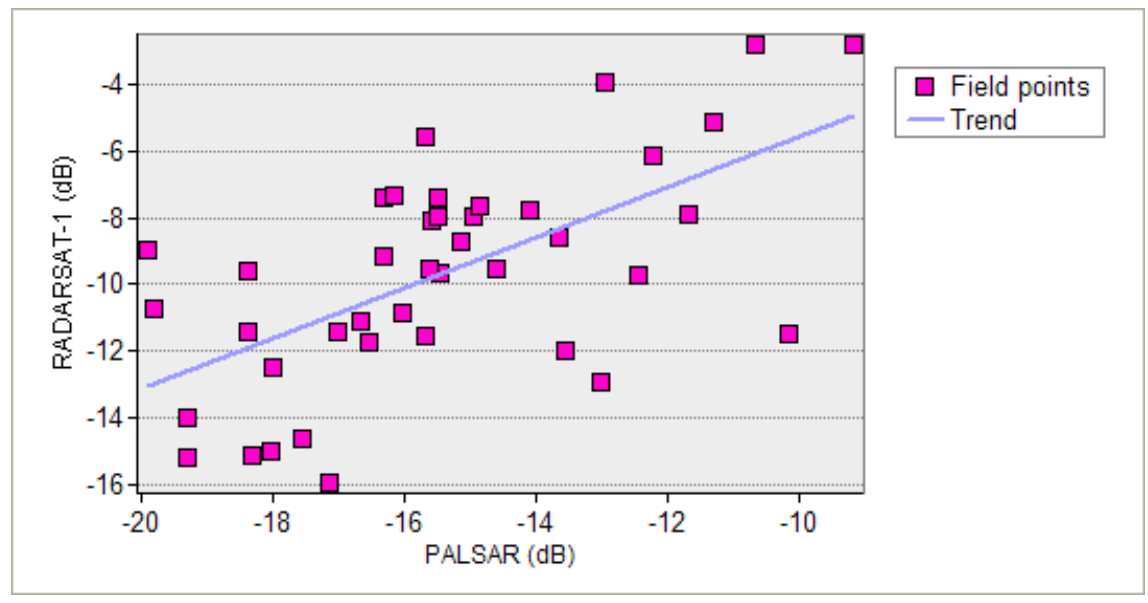

However, it was found that the ETM+/Radarsat-1 classification performed better in discriminating subtle textural differences throughout the alluvial area of Wadi Feiran than the ETM+/PALSAR classification. Two examples are shown in Figure 3 were selected wadi segments (A and B) are blown up to show differences in spatial class distribution and class categories as depicted in ETM+/Radarsat-1 (Figure 3a) and ETM+/PALSAR classifications (Figure 3b). They show that textural surface variability are better discriminated by Radarsat's shorter wavelengths (C-band) than PALSAR's longer wavelengths (L-band), which tends to show more homogenous areas with less contrasting class categories. PALSAR's greater spatial resolution, however, is able to pick up smaller spatial features and delineate sharper class boundaries.

An SRTM DEM of the study area was used to determine the slope as percent within the wadi channels to correlate this information with the classified hybrid images. The slope was classified into four classes based on which areas are favorable for water recharge (extremely flat areas) and which areas show high risks of flash floods (extremely steep areas) in the event of a rainstorm. The four classes are (Figure 5): flat terrain (0-1\%), gentle terrain (1-5\%), moderate terrain $(5-15 \%)$ and steep terrain $(>15 \%)$. The correlation results (Figure 6a) show that the smoothest surfaces with relatively small rock-fragment/sediment-grain sizes (classes 1, 2 and 3) mainly occur along gently sloping areas, whereas the roughest surfaces with large rock fragments and coarse grain sizes (classes 4 and 5) occur along the steepest sloping areas. This means that the most favorable areas for groundwater recharge are those covered with wadi deposits of fine to moderate grain sizes (fine sand to pebble; classes 1, 2 and 3$)$ and are located along relatively flat terrains $(0-1 \%)$, while areas with high risks of rock falls (erosion) and prone to flash floods are those wadi segments covered with very coarse-grained alluvial deposits (cobble to boulder; classes 4 and 5) and are located along moderate to steep terrains (>10\%) (Figure 9). 
Figure 8. Field photographs displaying the typical grain/fragment size of each hybrid image class from coarse to fine: (a) class 5-boulders, (b) class 4-boulders \& cobbles, (c) class 3-cobbles \& pebbles, (d) class 2-pebbles and sand, and (e \& f) class 1-sand and clay.
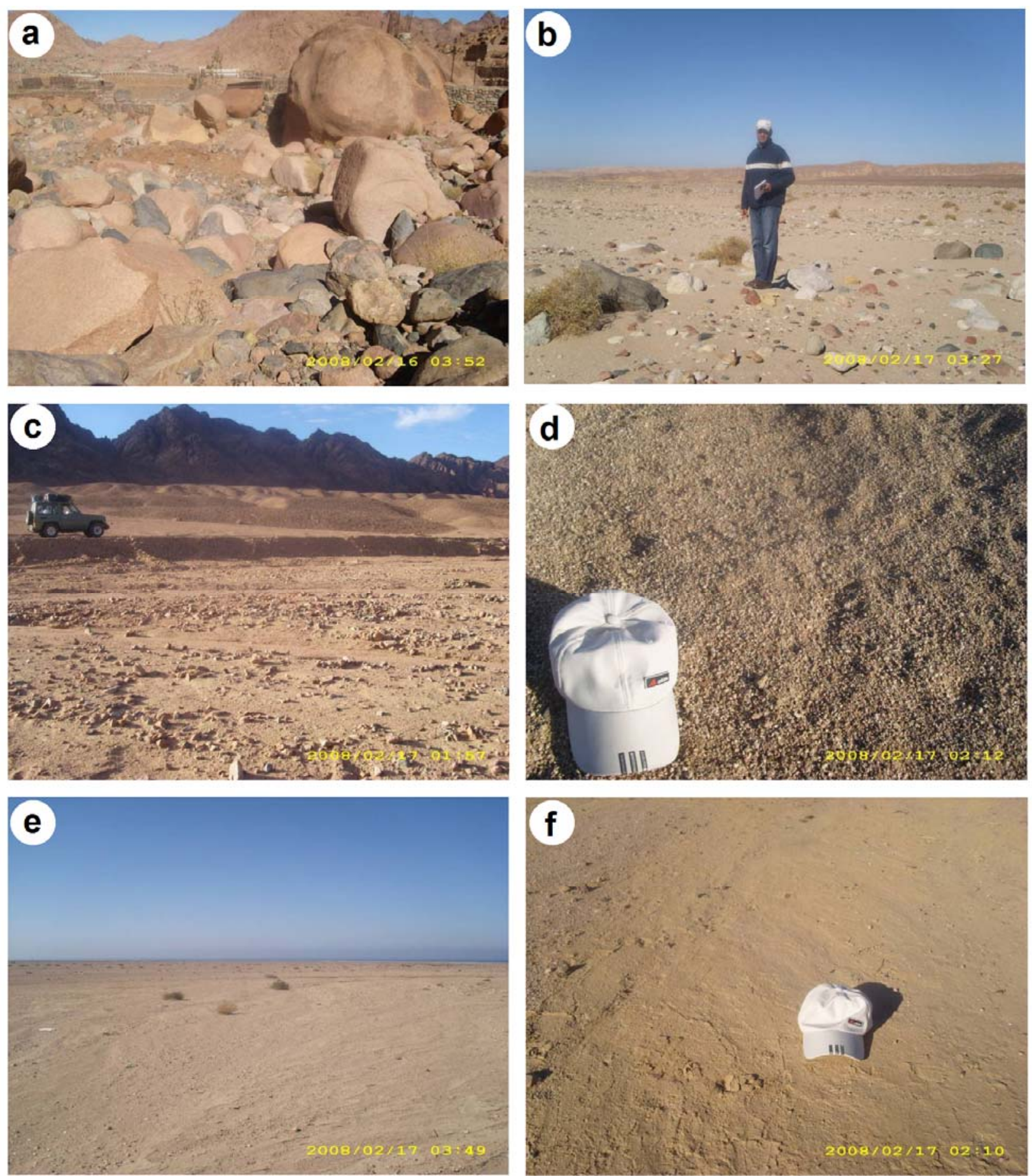
Figure 9. Promising areas along Wadi Feiran basin for (a) groundwater recharge, and (b) risky areas in the event of flash floods.
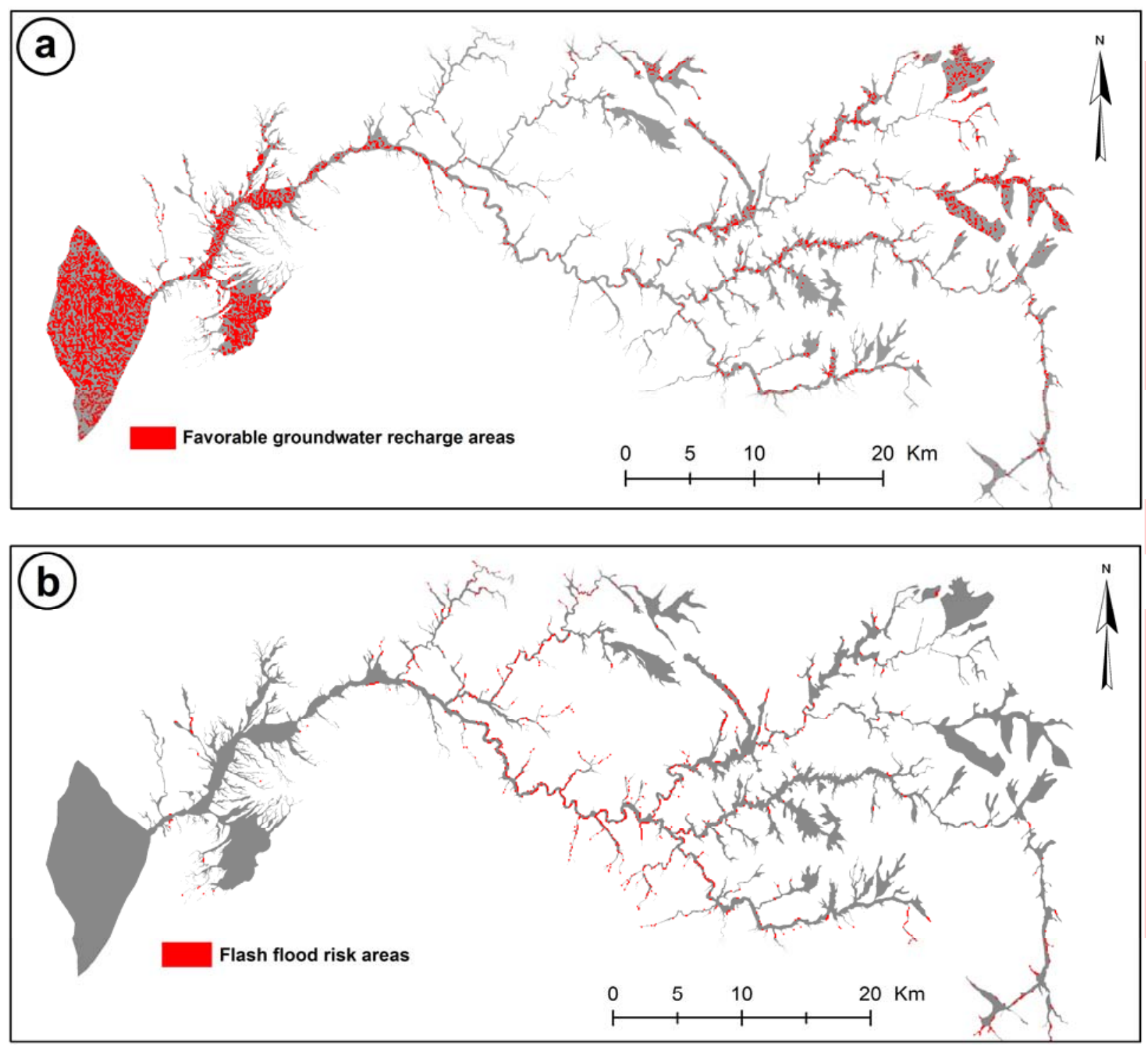

Results of this second correlation confirm that microwave surface measurements can be used as a proxy for slope measurements. This latter point was investigated in the field. In Figure 10 the mean backscatter coefficient values of Radarsat-1 (top) and PALSAR (bottom) at 41 sites surveyed in the field are plotted against SRTM slope values. All sites are located in relatively flat sections of the wadis away from the bedrock edges, which explains why the slope values range only from 0 to $10 \%$ (and not greater than 10\%). Wadi edges and smaller, narrower (and steeper) tributaries were excluded because of the layover effect that would have prevented obtaining reliable readings of backscatter coefficient values from the images. Both graphs show that with increasing backscatter and slope values, field points tend to be associated with higher class numbers of the respective hybrid image. This means that surface roughness increases even in less steep sections of the wadis. Another interesting observation in Figure 10 is that field points with higher backscatter values tend to be associated with higher class numbers, but in the case of Radarsat-1 even more so than in PALSAR. There are more field points marked as class 4 or 5 in the top graph (Radarsat-1) than in the bottom graph (PALSAR). The bottom 
graph shows that two thirds of the field points are classified in the ETM+/PALSAR data as classes 2 and 3. In other words Radarsat-1 seems to be more effective in differentiating smooth, intermediate and rough surfaces than PALSAR, and has therefore more discriminating power than the latter one.

Figure 10. Correlation of mean backscatter values (in $\mathrm{dB}$ ) of Radarsat-1 (A) and PALSAR (B) versus SRTM slope values (in \%) at selected sites visited in the field. Field locations are color coded according to their class membership. Note that the corresponding Radarsat-1 or PALSAR hybrid classification was used to assign class colors.
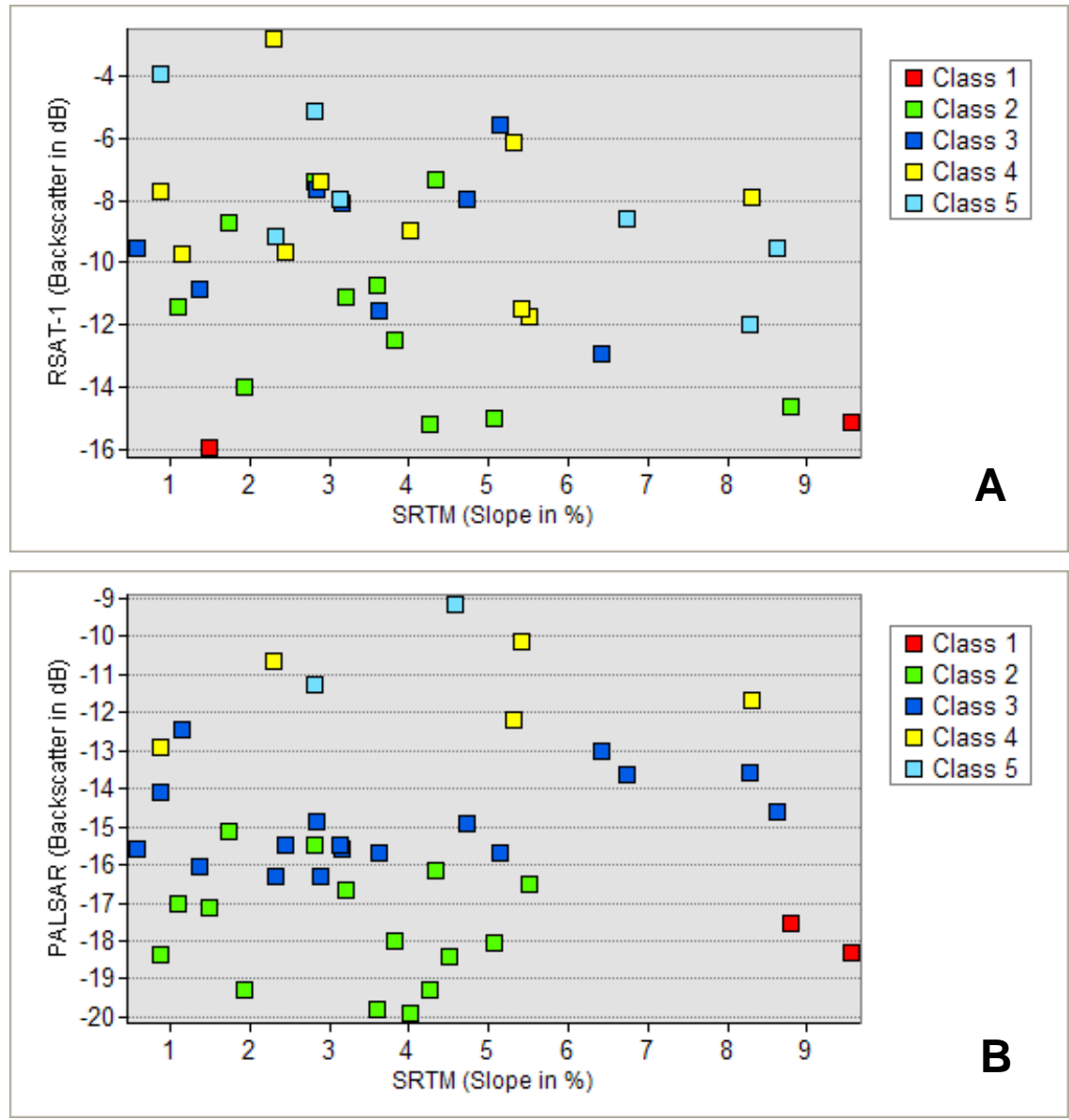

Thus, based on the above analysis, classes 1 to 5 of both hybrid images can be interpreted as representing decreased groundwater recharge potential and increased erosive stream power (Table 3). However, the Radarsat-1 hybrid image does a better job in discriminating micro-relief surfaces than the PALSAR hybrid image, which tends to assign smaller class numbers (smoother surface roughness classes) to wadi surfaces, missing, thus, to detect much of the micro-surface variations caused by stream erosion and deposition. 
Table 3. Relationships between radar backscatter, surface roughness, and slope, and its implication in terms of groundwater-recharge potential.

\begin{tabular}{|c|c|c|c|c|}
\hline $\begin{array}{l}\text { Class } \\
\text { (color) }\end{array}$ & $\begin{array}{c}\text { Radar } \\
\sigma 0 \text { values }\end{array}$ & $\begin{array}{l}\text { Roughness and } \\
\text { grain size }\end{array}$ & Slope & $\begin{array}{c}\text { Groundwater } \\
\text { recharge }\end{array}$ \\
\hline $\begin{array}{l}\text { Class } 1 \\
\text { (Red) }\end{array}$ & Low backscatter & Smooth (fine grains) & Gentle & High \\
\hline $\begin{array}{l}\text { Class } 2 \\
\text { (Green) }\end{array}$ & & & & 4 \\
\hline $\begin{array}{l}\text { Class } 3 \\
\text { (Blue) }\end{array}$ & To & To & To & To \\
\hline $\begin{array}{l}\text { Class } 4 \\
\text { (Yellow) }\end{array}$ & High backscatter & Rough (coarse grains) & Extreme & Low \\
\hline $\begin{array}{l}\text { Class } 5 \\
\text { (Cyan) }\end{array}$ & & & & \\
\hline
\end{tabular}

\subsection{Interpretation of Unmixing Results}

The output of the spectral unmixing procedure includes six fraction maps (one for each endmember) and an error image. The fraction maps show the spatial distribution of endmember abundances, and the error image is a measure of how well the endmember proportions fit the mixing model. If the error image does not show any systematic patterns, and mainly contains random noise, then the model fits perfectly. This is the case with the results obtained in this work. Moreover, the resulting fraction maps should have a value range from 0 to 1 , where 0 means no matching between the endmember spectra and the derived spectra from pixel unmixing and 1 means a complete match. Sometimes, the values range from $<0$ and $>1$, especially for the unconstrained unmixing, due to fraction overflow and residual increase [22]. Table 4 displays the statistics of the five endmember-fraction and error images.

The spatial distribution of the carbonate fraction coincides with the sedimentary rock unit (mainly limestone) of the simplified geologic map. At some places it is covered by weathered old granite (Figure 11b). These weathered old granites most probably were covered by a thin sedimentary cap like the much closer granites on the northeastern border of the Feiran basin. This sedimentary cap was washed out by torrential rainfalls and deposited within the wadi channels [6]. The spectral reflectance curves of the old granite and volcanic endmembers are quite similar (Figure 4). Thus, their fraction maps show very similar spatial distributions within the wadi courses (Figure 11b,e). They are predominantly found in areas that are wide, relatively flat and surrounded by weathered old granite and volcanics. The young granite fraction is widely distributed along the wadi channel (Figure 11c) because it is located at the most distant and highest terrain portion of the basin (i.e., very long travel distance to the basin outlet) and has a relatively light color and well represented endmember spectrum. Conversely, the metamorphic fraction is grey to dark in color and represents the lowest spectral reflectance values (Figure 11d). The colors in the fraction maps range from black to white, with lighter colors representing high fraction values and darker colors representing low fraction values. However, in the case of the residual error map high error values are shown with light colors and low errors with dark colors (Figure 11f). 
Table 4. Statistics of the five endmember fraction and RMS error images of the alluvial area in Wadi Feiran basin. Bedrock outcrops and vegetation were masked out.

\begin{tabular}{lcccccc}
\hline \multirow{2}{*}{ ETM } & \multicolumn{7}{c}{ Unmixing Fractions } \\
\cline { 2 - 7 } & Carbonate & Old granite & Young granite & Metamorphic & Volcanic & RMS error \\
\hline Min Value & -0.177591 & -3.296668 & -0.866585 & -10.176119 & -5.59556 & 0.00000 \\
Max Value & 1.457814 & 2.680196 & 2.792672 & 7.578426 & 4.158999 & 0.007072 \\
Mean & 0.231171 & 0.059169 & 0.528635 & -0.76337 & -0.17111 & 0.000606 \\
1 Stdev. & 0.091271 & 0.386858 & 0.263685 & 1.402204 & 0.735009 & 0.000459 \\
Percent $<0$ & 0.3 & 50.5 & 3.2 & 73.7 & 66.6 & 3.0 \\
Percent $0-1$ & 99.7 & 47.7 & 94.4 & 19.2 & 28.3 & 97.0 \\
Percent $>1$ & 0.0 & 1.8 & 2.4 & 7.1 & 5.1 & 0.0 \\
\hline
\end{tabular}

A density slicing was applied to each faction map using the following intervals: $(<0.0),(0.0-0.25)$, $(0.25-0.5),(0.5-0.75),(0.75-1.0)$ and $(>1)$; this means that the fraction percentage within the pixel is ( $<0 \%),(0 \%$ to $25 \%),(25 \%$ to $50 \%),(50 \%$ to $75 \%),(75 \%$ to $100 \%)$ and (s $>100 \%)$, respectively. The low-end values $(<0 \%)$ are added to the $0 \%$ to $25 \%$ interval and the high-end values $(>100 \%)$ are added to $75 \%$ to $100 \%$. The final intervals are $0 \%$ to $25 \%$ (low predominance), $25 \%$ to $50 \%$ (low to medium predominance), $50 \%$ to $75 \%$ (medium to high predominance) and $75 \%$ to $100 \%$ (high predominance).

The same method used for correlating the classified hybrid images with the slope was applied to correlate the classified ETM+/Radarsat-1 hybrid image with the fraction maps. The results of this correlation are presented in figure 6 and summarized in Table 5 where predominant endmembers within each class of the fused image are highlighted. In addition, Table 6 displays the total percentage of endmember fractions per hybrid classes. From these tables and the graphs in figure 6 the following conclusions can be drawn: (1) the young granite fraction is the most predominant endmember and appears in all classes with relatively high percentages, while the old granite is only marginally present with slightly increased percentages in classes 4 and 5; (2) conversely, the metamorphic and volcanic fractions appear in small amounts in all classes; (3) followed by slightly larger amounts of the carbonate fraction, especially in classes 2 and 3.

Comparison of the spatial distribution of endmembers (Figure 10) with the geological map (Figure 1) shows the location of the host rocks with respect to the wadi deposits. The carbonate fractions are mostly present at the lower west portion of the basin not far from their sedimentary source-rock area; whereas the source-rock area of the younger granite fraction is in the upper east portion of the basin far away from the basin outlet. The fact that this endmember fraction is present all along the entire wadi system, shows its greater resistance and longer travel distance with respect to other endmembers. Not surprisingly the young granite is the most predominant endmember fraction in all classes. The distribution of the metamorphic fraction coincides with the location of its source rock and is present in the alluvial fan area. However, the metamorphic as well as the volcanic and old granite endmembers appear in relatively small amounts with respect to the young granites and carbonates. 
Table 5. Predominant rock composition (endmembers) within each class produced by unsupervised classification of the hybrid image.

\begin{tabular}{ccccccc}
\hline $\begin{array}{c}\text { Class } \\
\text { s }\end{array}$ & Carbonate & $\begin{array}{c}\text { Old } \\
\text { granite }\end{array}$ & $\begin{array}{c}\text { Young } \\
\text { granite }\end{array}$ & Metamorphic & Volcanic & $\begin{array}{c}\text { Predominant } \\
\text { EM }\end{array}$ \\
\hline 1 & $\mathbf{X X X}$ & $\mathbf{X X}$ & $\mathbf{X X X}$ & $\mathbf{X}$ & $\mathbf{X X}$ & $\begin{array}{l}\text { Young Granite } \\
\text { and Carbonate } \\
\text { Young }\end{array}$ \\
\hline 2 & $\mathbf{X X X X}$ & $\mathbf{X}$ & $\mathbf{X X X X}$ & $\mathbf{X X X}$ & $\mathbf{X}$ & $\begin{array}{l}\text { Granite, } \\
\text { Carbonate and } \\
\text { Metamorphic } \\
\text { Carbonate }\end{array}$ \\
3 & $\mathbf{X X X X}$ & $\mathbf{X X}$ & $\mathbf{X X X}$ & $\mathbf{X X X}$ & $\mathbf{X}$ & $\begin{array}{l}\text { Young Granite } \\
\text { and } \\
\text { Metamorphic }\end{array}$ \\
\hline 4 & $\mathbf{X X}$ & $\mathbf{X X X X}$ & $\mathbf{X X}$ & $\mathbf{X X}$ & $\mathbf{X X X}$ & $\begin{array}{l}\text { Old Granite } \\
\text { and Volcanic }\end{array}$ \\
5 & $\mathbf{X}$ & $\mathbf{X X X}$ & $\mathbf{X}$ & $\mathbf{X X X X}$ & $\mathbf{X X X X}$ & $\begin{array}{l}\text { Metamorphic } \\
\text { and Volcanic }\end{array}$ \\
\hline
\end{tabular}

Note: $\mathrm{X}=$ Low, $\mathrm{XX}=$ Low to Medium, $\mathrm{XXX}=$ Medium to High, $\mathrm{XXXX}=$ High and $\mathrm{EM}=$ Endmember

Table 6. Statistical distribution of endmember fractions per hybrid classes. Numbers are in percent.

\begin{tabular}{ccccccccccccccccccccc}
\hline \multirow{2}{*}{ Classes } & \multicolumn{4}{c}{ Old granite } & \multicolumn{1}{c}{ Young granite } & \multicolumn{4}{c}{ Metamorphic } & \multicolumn{4}{c}{ Volcanic } & \multicolumn{4}{c}{ Carbonate } \\
& L & M-L & M-H & H & L & M-L & M-H & H & L & M-L & M-H & H & L & M-L & M-H & H & L & M-L & M-H & H \\
\hline $\mathbf{1}$ & 74 & 16 & 7 & 3 & 11 & 29 & 38 & 22 & 80 & 5 & 5 & 10 & 74 & 9 & 8 & 9 & 62 & 38 & 0 & 0 \\
$\mathbf{2}$ & 76 & 14 & 7 & 3 & 10 & 28 & 37 & 25 & 78 & 6 & 4 & 12 & 79 & 9 & 5 & 7 & 50 & 50 & 0 & 0 \\
$\mathbf{3}$ & 73 & 15 & 7 & 5 & 12 & 27 & 38 & 23 & 77 & 6 & 4 & 13 & 79 & 9 & 4 & 8 & 50 & 50 & 0 & 0 \\
$\mathbf{4}$ & 66 & 17 & 10 & 7 & 18 & 36 & 34 & 12 & 78 & 7 & 3 & 12 & 71 & 11 & 8 & 10 & 64 & 32 & 0 & 0 \\
$\mathbf{5}$ & 70 & 16 & 9 & 5 & 21 & 42 & 28 & 9 & 76 & 8 & 4 & 12 & 69 & 11 & 8 & 10 & 79 & 21 & 0 & 0 \\
\hline
\end{tabular}

Note: $\mathrm{L}=$ Low, $\mathrm{M}-\mathrm{L}=$ Medium to Low, $\mathrm{M}-\mathrm{H}=$ Medium to High and $\mathrm{H}=$ High

Field verification carried out in February 2008 confirmed the results of the image processing work and shows that the interpretation of the resulting products matches field observation on grain/fragment size distribution and the predominant rock composition within each class (Figure 8). Furthermore, the backscatter coefficient measurements obtained at the surveyed field points and their relationship to rock type and fragment/sediment grain size is comparable to other similar studies conducted in arid environments, specifically that of [20] who demonstrated a close relationship between backscatter coefficient and the maximum height of rocks in fluvial and aeolian deposits of the western Sahara desert. 
Figure 11. Fraction maps of image endmembers (lighter colors mean high fraction values and dark colors mean low fraction values; except for the error map, where high error values are shown in white and low error values in black). (a) Carbonate, (b) Old granite, (c) Young granite, (d) Metamorphic, (e) Volcanic and (f) RMS error of unmixing result.
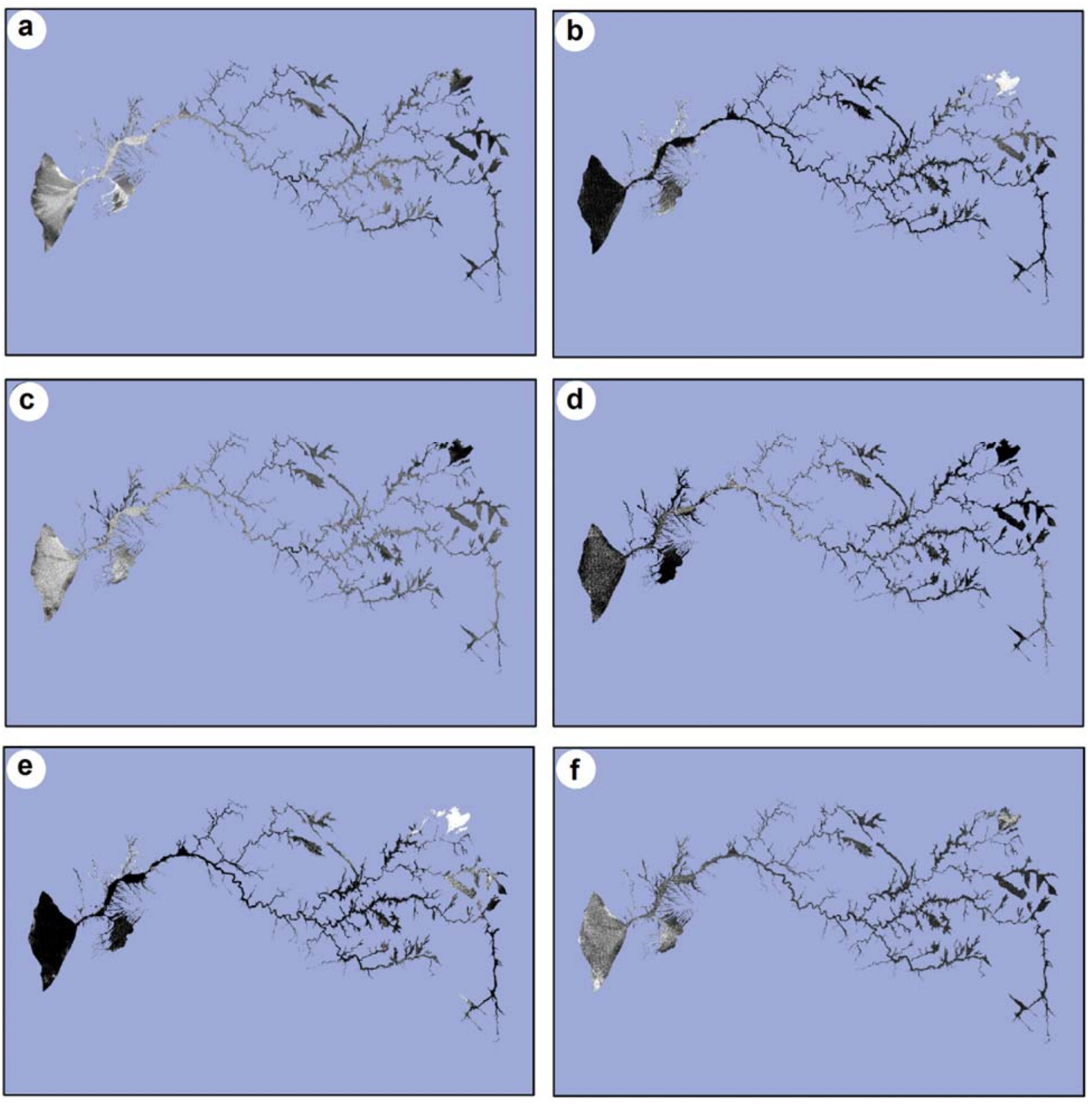

\section{Conclusions}

Water is a scarce and much needed resource in arid lands. In the Sinai Peninsula of Egypt the study of ephemeral watercourses (wadis) is of great importance because of their potential role in supplying water resources. In the event of torrential rainfalls, large quantities of surface water may accumulate in these wadis, producing two different effects, namely flash floods and groundwater recharge.

Two standard image processing methods were used to map and characterize Wadi Feiran deposits using three types of satellite images. In the first method, an optical (Landsat ETM+) image was fused 
with two sets of microwave images (Radarsat-1 and PALSAR) with different spatial and spectral characteristics. The resulting hybrid images were classified in terms of spectral and textural properties of the alluvial wadi floor materials. Both classifications show slightly different class distributions, with Radarsat-1 hybrid-classes matching more closely field-observed textural variations in wadi surfaces. This suggests that the shorter wavelength of Radarsat-1 (C-band) enables better detection of subtle textural variations than the longer wavelength of PALSAR (L-band). However, class boundaries are better defined in the fine beam $(6.25 \mathrm{~m})$ PALSAR data than in the standard beam $(12.5 \mathrm{~m})$ Radarsat-1 data.

In the second method, the spectral mixture modeling is performed on a Landsat ETM+ image to identify the source material (endmembers) and the predominant rock composition of wadi deposits. A correlation between the endmember fractions and the ETM+/Radarsat-1 hybrid classes shows that none of the endmembers occur in large proportions $(>50 \%)$ within the wadi deposits. The most resistant rock type is the young granite unit which crops out in the upper portion of the basin. It has, thus, the longest travel distance to the basin outlet compared to the other rock units. The unmixing results show that the young granite endmember has the highest fraction in the wadi deposits along the entire wadi system, indicating that the most active streams are those located in the southeastern upper portion of the basin where the source of the granite is located. In contrast, the old granite unit makes up large parts of the middle-upper basin but appears in relatively small fraction amounts in the wadi deposits. Geomorphologically, the landscape were the young granites crop out is quite different from that of the old granites; meaning that the former is more rugged with narrower and deeper incised wadis than the latter. Erosive processes are, therefore, currently more active in the young granite source area, which explains its relatively high presence in wadi deposits from the head waters down to the alluvial fan area.

This research has demonstrated that the textural and compositional characteristics of wadi deposits can be reliably mapped using data fusion classification and linear spectral mixture analysis. This information is used to identify the favorable areas for groundwater recharge and areas at risk of flash flood events along Wadi Feiran basin.

\section{Acknowledgements}

The authors would like to thank the Japan Aerospace Exploration Agency (JAXA) for providing the ALOS-PALSAR images as part of the ALOS user agreement (ALOS-RA-81), as well as the King Abdul Aziz City for Science and Technology (KACST) facility in Saudi Arabia for providing the Radarsat-1 image.

\section{References and Notes}

1. Issar, A.; Bruins, H. Special climatological conditions in the deserts of Sinai and the Negev during the latest Pleistocene. Palaeogeogr. Palaeoclimatol. 1983, 43, 63-72.

2. El-Baz, F.; Himida, I. Recommendations for the strategy of groundwater exploration in the deserts of Egypt. In Ground Water Potential of the Sinai Peninsula, Egypt; El-Baz, F., Kusky, T., Himida, I., Mogheeth, A., Eds.; Ministry of Agriculture and Land Reclamation: Cairo, Egypt, 1998; pp. 217-219. 
3. Gaber, A.; Ghoneim, E.; Khalaf, F.; El-Baz, F. Delineation of paleolakes in the Sinai Peninsula, Egypt, using remote sensing and GIS. J. Arid Environ. 2009, 73, 127-134.

4. Koch, M.; Blanco-Ward, D.; El-Baz, F. Characterizing active wadi channels in arid lands by linear mixture modeling. SPIE 1999, 3868, 261-270.

5. Koch, M.; El-Baz, F. Spectral and textural classification of active wadi systems in arid lands by Landsat TM and Radarsat data. In CDROM-Proceedings of the ASPRS Annual Conference, Washington, DC, USA, May, 2000; p. 10.

6. Geological Map of South Sinai, Egypt, 1987, Scale 1: 500 000; The Egyptian General Petroleum Corporation: Cairo, Egypt, 1987.

7. Mather, P.M.; Brandt, T.; Koch, M. An evaluation of Landsat TM spectral data and SAR-derived textural information for lithological discrimination in the Red Sea Hills, Sudan. Int. J. Remote Sens. 1998, 19, 587-604.

8. Welch, R.; Ahlers, W. Merging multiresolution SPOT HRV and Landsat TM data. Photogramm. Eng. Remote Sens. 1987, 53, 301-303.

9. ENVI (Environment for Visualizing Images); ITT Visual Information Solutions: Boulder, CO, USA, 2008.

10. Xie, H.; Keller, G.R. Fusion of Landsat ETM+ and radar data to enhance the extraction of surface and near-surface information. Geol. Soc. Amer. 2006, 397, 141-151.

11. Pohl, C.; van Genderen, J.L. Multisensor image fusion in remote sensing: concepts, methods and applications. Int. J. Remote Sens. 1998, 19, 823-854.

12. Leconte, R.; Brissette, F.; Galarneau, M.; Rousselle, J. Mapping near-surface soil moisture with RADARSAT-1 synthetic aperture radar data. Water Resour. Res. 2004, 40, 1-13.

13. Stearns, S.V.; van der Horst, E.; Swihart, G. Hyperspectral mapping of borate minerals in Death Valley, California. In Proceedings of the Thematic Conference on Geologic Remote Sensing, Vancouver, Canada, 1999; pp. 81-85.

14. Hubbard, B.E.; Crowley, J.K. Mineral mapping on the Chilean-Bolivian Altiplano using co-orbital ALI, ASTER and hyperion imagery: data dimensionality issues and solutions. Remote Sens. Environ. 2005, 99, 173-186.

15. Metternicht, G.I.; Fermont, A. Estimating erosion surface features by linear mixture modeling. Remote Sens. Environ. 1998, 64, 254-265.

16. Koch, M. Geological controls of land degradation as detected by remote sensing: A case study in Los Monegros, NE Spain. Int. J. Remote Sens. 2000, 21, 457-473.

17. Green, A.A.; Berman, M.; Switzer, P.; Craig, M.D. A transformation for ordering multispectral data in terms of image quality with implications for noise removal. IEEE Trans. Geosci. Remot. Sen. 1988, 26, 65-74.

18. Boardman, J.W.; Kruse, F.A.; Green, R.O. Mapping target signatures via partial unmixing of AVIRIS data. In Summaries of Fifth JPL Airborne Earth Science Workshop; JPL Publication 95-1: Pasadena, CA, USA, 1995; pp. 23-26.

19. MapReady; Alaska Satellite Facility (ASF): Geophysical Institute of the University of Alaska: Fairbanks, AK, USA, 2009. 
20. Deroin, J.P.; Company, A.; Simonin, A. An empirical model for interpreting the relationship between backscattering and arid land surface roughness as seen with the SAR. IEEE Trans. Geosci. Remot. Sen. 1997, 35, 86-92.

21. Mather, P.M. Computer Processing of Remotely-Sensed Images: an Introduction, 3rd ed.; John Wiley \& Sons: Chichester, UK, 2004.

22. Adams, J.B.; Gillespie, A.R. Remote Sensing of Landscapes with Spectral Images: a Physical Modeling Approach; Cambridge University Press: Cambridge, UK, 2006; pp. 126-183.

(C) 2010 by the authors; licensee Molecular Diversity Preservation International, Basel, Switzerland. This article is an open-access article distributed under the terms and conditions of the Creative Commons Attribution license (http://creativecommons.org/licenses/by/3.0/). 